Key words: nonlinear rotation, viscoelastic, functionally graded, successive approximations, solid and annular disks, gradually varying thickness

\title{
ELASTIC AND VISCOELASTIC STRESSES OF NONLINEAR ROTATING FUNCTIONALLY GRADED SOLID AND ANNULAR DISKS WITH GRADUALLY VARYING THICKNESS
}

\begin{abstract}
Analytical and numerical nonlinear solutions for rotating variable-thickness functionally graded solid and annular disks with viscoelastic orthotropic material properties are presented by using the method of successive approximations. Variable material properties such as Young's moduli, density and thickness of the disk, are first introduced to obtain the governing equation. As a second step, the method of successive approximations is proposed to get the nonlinear solution of the problem. In the third step, the method of effective moduli is deduced to reduce the problem to the corresponding one of a homogeneous but anisotropic material. The results of viscoelastic stresses and radial displacement are obtained for annular and solid disks of different profiles and graphically illustrated. The calculated results are compared and the effects due to many parameters are discussed.
\end{abstract}

\section{Nomeclature}

$\begin{array}{ll}r & \text { radial direction } \\ u & \text { radial displacement } \\ \sigma_{r}, \sigma_{\theta} & \text { radial and circumferential stresses } \\ \rho & \text { material density } \\ \Omega & \text { angular velocity } \\ \varepsilon_{r}, \varepsilon_{\theta} & \text { radial and circumferential strains }\end{array}$

\footnotetext{
${ }^{1}$ Department of Mathematics, Faculty of Science, Mansoura University, Mansoura 35516, Egypt.

${ }^{2}$ Department of Mathematics, Faculty of Science, Damietta University, Damietta 34517, Egypt.

${ }^{3}$ Department of Mathematics, Faculty of Science, King Abdulaziz University, P.O. Box 80203, Jeddah 21589, Saudi Arabia. Email: zenkour@kau.edu.sa.

${ }^{4}$ Department of Mathematics, Faculty of Science, Kafrelsheikh University, Kafrelsheikh 33516, Egypt.Email:zenkour@sci.kfs.edu.eg.
} 


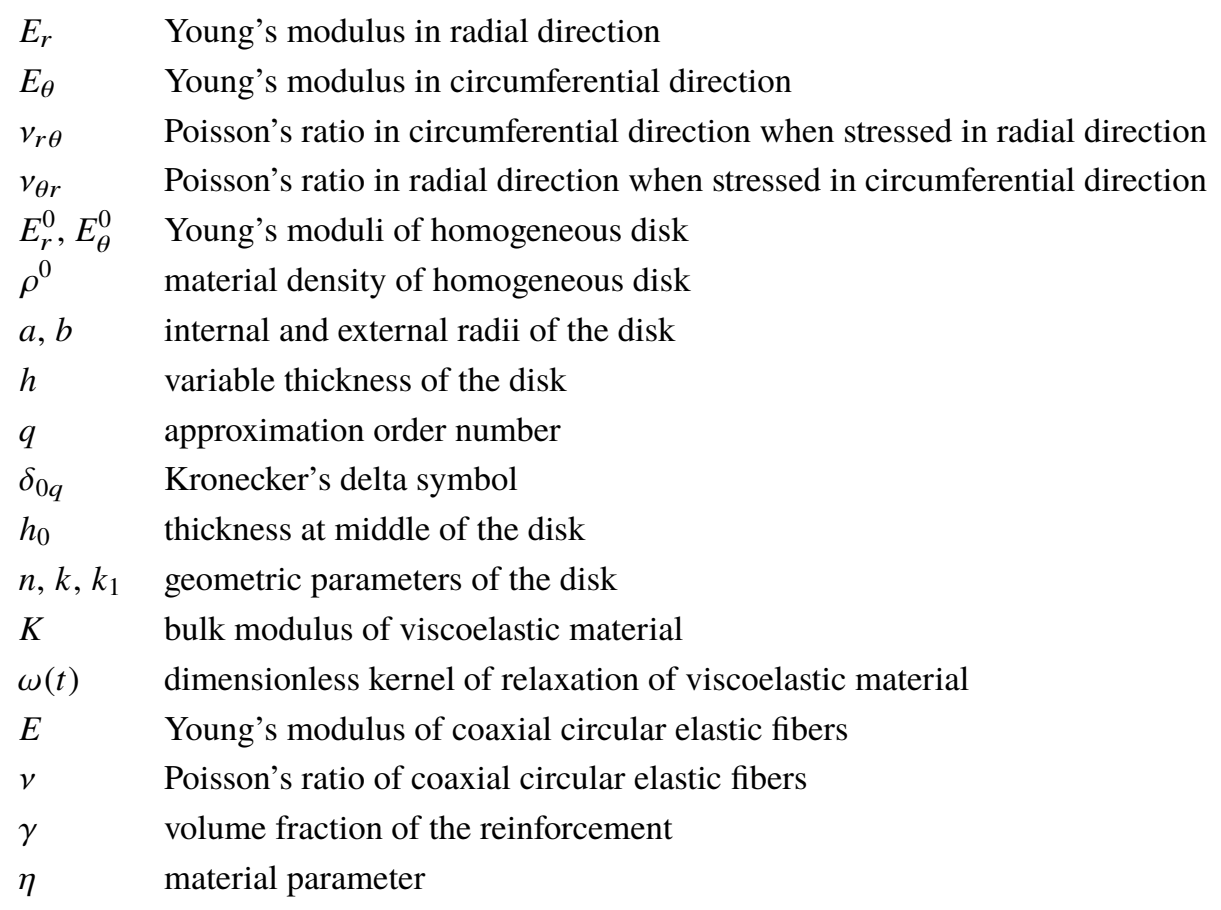

\section{Introduction}

Rotation of solid and annular disks are of practical concern in many aerospace industries including gas turbines, aircraft parts, rockets, and in many fields of engineering such as marine and mechanical applications. One of the famous examples of rotating solid disks is the brake disk in which only body forces are involved. Most applications of the solid and annular disk are concerned with their rotation, the thermal or thermomechanical loads applied, and their linearity or nonlinearity forms. In the linear case, investigators mainly used infinitesimal elasticity theory to study the isotropic or anisotropic disks of uniform-thickness profiles [1]. However, in nonlinear analysis, the scientific literature mainly focused on material and analysis as well as the nonlinear geometry. The applications of non-uniform, variable-thickness disks are rapidly increasing due to economic considerations and to improve the mechanical performances [2]. Eraslan and Orcan [3] and Eraslan and Argeso [4] dealt with deformation of rotating variable-thickness disks. They found that, at the same angular velocity, the stresses for both rotating variable-thickness solid and annular disks have the smallest values in comparison with those of the uniform-thickness disks.

Timoshenko and Goodier [5] dealt with different problems of the deformation of rotating disks. These disks may be made of linear elastic, isotropic and homogeneous materials. However, Horgan and Chan [6] studied the behavior of disks which were composed of functionally graded materials. They assumed that 
Young's modulus $E$ is functionally graded (FG) in the radial direction as a powerlaw function of the radius $r$ of the disk. Jahed et al. [7] and Hojjati and Hassani [8] took into consideration the temperature-dependent material properties to present a procedure for minimum mass design of rotating disks. They dealt with the rotating disks with variable material properties at a high temperature. Eraslan and Akis [9] assumed that Young's modulus $E$ may change in the radial direction as an exponential or a parabolic function of $r$. They obtained closed-form solutions for rotating FG solid shafts and disks. Kordkheili and Naghdabadi [10] studied the axisymmetric thermoelastic deformations of rotating FG hollow and solid disks by using a semi-analytical approach. They assumed that the thermomechanical properties are changed according to a power-law function of $r$ in the radial direction. You et al. [11] assumed that Young's modulus, thermal expansion coefficient, and mass density are all varying in the radial direction of the disk according to power-law functions of $r$. They considered the disk is to be subjected to a uniform temperature change to derive the closed-form solutions of such rotating FG disks. Hojjati and Jafari [12] assumed that the rotating annular elastic disks may be of uniform or variable thicknesses and mass densities. They used both the homotopy perturbation and Adomian's decomposition analytical methods to find stresses and displacements in such disks. Vullo and Vivio [13, 14] discussed the rotating annular and solid disks with variable thickness and density. They investigated the constitutive relations of stresses and strains in the thermally loaded rotating variable-thickness elastic disks. Bayat et al. [15-18] dealt with two problems, the elastic and thermoelastic for rotating FG disks at steady temperature. They assumed that the material properties and thickness of the disk are considered according power-law functions of $r$. Zenkour $[19,20]$ studied the stress distribution in rotating sandwich solid disks with a FG core and isotropic face sheets. Nie and Batra [21] presented the axisymmetric deformations of rotating variable thickness disk. Beside the variable-thickness, they assumed that the mass density, thermal expansion coefficient, and shear modulus of the disk are varying in the radial direction.

The rotating viscoelastic solid and annular disks is of interest in the design of many applications. Gears, pulleys and computer storage disks are considered as examples of viscoelastic components. Feng [22] presented either elastic or viscoelastic deformations of a rotating disk. He obtained the governing equations in terms of two coupled first-order ordinary differential-integral equations with explicit derivatives. Zenkour and Allam [23] developed analytical elastic and viscoelastic solutions for the deformation of elastic rotating variable-thickness disks. They assumed that the disks are made of orthotropic fiber-reinforced viscoelastic composite materials with equal or different Young's moduli. Allam et al. [24] discussed the viscoelastic deformation of rotating variable thickness solid and annular disks. They dealt with the analytical solutions and assumed that the disks are made of inhomogeneous viscoelastic orthotropic materials. Zenkour [25-29] dealt with different problems of thermoelastic analysis of annular and solid sandwich or FGM disks with arbitrary variable thickness. 
The aim of this article is to discuss different problems of rotating FG disks. It presents different solutions for fully elastic or viscoelastic inhomogeneous deformation of rotating FG nonlinear circular solid and annular disks with various forms of variable-thickness variations. The thickness, density and moduli of elasticity are considered as functions of the radial direction according to different power-laws. Radial displacement and hoop and radial stresses of the rotating disks are graphically illustrated. A comparison between the behavior of solid and annular disk is presented and discussions about the results are made.

\section{Basic equations}

Figure 1 shows a circular disk with internal and external radii $a$ and $b$, its profile varies in the radial direction in arbitrary law $h(r)$, where $r$ is the radial coordinate. It is assumed that plane stress assumption is justified since the thickness of the disk is much smaller than its diameter. The effect of thickness variation of rotating disks is taken under consideration to get reliable results as in the case of uniformthickness disks. That is because the theory of the variable-thickness disks gives excellent results as long as it meets the assumptions of plane stress. So, the equation of motion of rotating variable thickness and density is expressed as

$$
\frac{1}{r} \frac{\mathrm{d}}{\mathrm{d} r}\left(h r \sigma_{r}\right)-\frac{h \sigma_{\theta}}{r}+h \rho \Omega^{2} r=0 .
$$

The nonlinear Cauchy's relations are written as

$$
\varepsilon_{r}=\frac{\mathrm{d} u}{\mathrm{~d} r}+\frac{1}{2}\left(\frac{\mathrm{d} u}{\mathrm{~d} r}\right)^{2}, \quad \varepsilon_{\theta}=\frac{u}{r} .
$$

The constitutive relations of an orthotropic disk can be described in forms of the elastic deformation as

$$
\varepsilon_{r}=\frac{1}{E_{r}} \sigma_{r}-\frac{v_{\theta r}}{E_{\theta}} \sigma_{\theta}, \quad \varepsilon_{\theta}=-\frac{v_{r} \theta}{E_{r}} \sigma_{r}+\frac{1}{E_{\theta}} \sigma_{\theta} .
$$

Substitution of Eq. (2) into Eq. (3) gives the constitutive equations for the radial and circumferential stresses as:

$$
\begin{gathered}
\sigma_{r}=\frac{E_{r}}{1-v_{r \theta} v_{\theta r}}\left[\frac{\mathrm{d} u}{\mathrm{~d} r}+\frac{1}{2}\left(\frac{\mathrm{d} u}{\mathrm{~d} r}\right)^{2}+v_{\theta r} \frac{u}{r}\right], \\
\sigma_{\theta}=\frac{E_{\theta}}{1-v_{r \theta} v_{\theta r}}\left[v_{r \theta}\left(\frac{\mathrm{d} u}{\mathrm{~d} r}+\frac{1}{2}\left(\frac{\mathrm{d} u}{\mathrm{~d} r}\right)^{2}\right)+\frac{u}{r}\right] .
\end{gathered}
$$

Here, the FG material properties of the disk are classified as

$$
\left\{E_{r}, E_{\theta}, \rho\right\}=F(r)\left\{E_{r}^{0}, E_{\theta}^{0}, \rho^{0}\right\} .
$$


It is to be noted that for orthotropic, homogeneous disks the following relation between ratios and moduli should be satisfied:

$$
v_{r} E_{\theta}^{0}=v_{\theta r} E_{r}^{0}
$$

\section{Formulation of elastic solution}

The stress components appearing in Eq. (4) may be used in the Eq. (1) with the help of the material properties appearing in Eq. (5) to get the following nonlinear differential equation:

$$
\frac{\mathrm{d}^{2} u}{\mathrm{~d} r^{2}}+G(r) \frac{\mathrm{d} u}{\mathrm{~d} r}+v_{\theta r}\left[G(r)-\frac{1}{v_{r} \theta}-1\right] \frac{u}{r^{2}}+H(r)=0,
$$

where

$$
\begin{gathered}
H(r)=\frac{\mathrm{d}^{2} u}{\mathrm{~d} r^{2}} \frac{\mathrm{d} u}{\mathrm{~d} r}+\frac{G(r)-v_{\theta r}}{2 r}\left(\frac{\mathrm{d} u}{\mathrm{~d} r}\right)^{2}+\frac{\rho \Omega^{2}\left(1-v_{r \theta} v_{\theta r}\right) r}{2 E_{r}^{0}}, \\
G(r)=\frac{1}{h(r) F(r)} \frac{\mathrm{d}}{\mathrm{d} r}[r h(r) F(r)] .
\end{gathered}
$$

Now, we consider the boundary conditions for annular disk as

$$
\left.\sigma_{r}\right|_{r=a}=\left.\sigma_{r}\right|_{r=b}=0
$$

while those for solid disk are considered to be

$$
\sigma_{r} \text { at } r=0 \text { is finite and }\left.\sigma_{r}\right|_{r=b}=0 .
$$

In what follows, we will introduce the dimensionless $\bar{r}=r / b$ in the above equations, and drop the bar for simplicity.

\subsection{Successive approximation method}

The nonlinear differential equation appearing in Eq. (7) may be constructed as a system of linear differential equations. Successive approximation method is applied to solve the corresponding linear problem (a system of differential equations) as follows:

Let us construct the following system of linear differential equations for $q=$ $0,1,2,3, \ldots$

$$
\frac{\mathrm{d}^{2} u_{q}}{\mathrm{~d} r^{2}}+G(r) \frac{\mathrm{d} u_{q}}{\mathrm{~d} r}+\frac{v_{\theta r}}{r^{2}}\left[G(r)-\frac{1}{v_{r \theta}}-1\right] u_{q}+H_{q}(r)=0,
$$

where $q$ is the approximation order number, and the boundary conditions become

Annular disk: $\left.\sigma_{r}^{q}\right|_{r=s}=\left.\sigma_{r}^{q}\right|_{r=1}=0, \quad s=\frac{a}{b}$,

Solid disk: $\sigma_{r}^{q}$ at $r=0$ is finite and $\left.\sigma_{r=1}^{q}\right|_{r=1}=0$. 
Also

$$
H_{q}(r)=H_{0}(r)+\left[\frac{\mathrm{d}^{2} u_{q-1}}{\mathrm{~d} r^{2}} \frac{\mathrm{d} u_{q-1}}{\mathrm{~d} r}+\frac{G(r)-v_{\theta r}}{2 r}\left(\frac{\mathrm{d} u_{q-1}}{\mathrm{~d} r}\right)^{2}\right]\left(1-\delta_{0 q}\right),
$$

in which $\delta_{0 q}$ is the Kronecker's delta symbol and

$$
H_{0}(r)=\frac{\rho^{0} \Omega^{2}\left(1-v_{r \theta} v_{\theta r}\right) r}{2 E_{r}^{0}} .
$$

We notice that when $q=0$ (the zero solution), we have the same solution as the well-known in the linear case [5].

The general solutions for linear differential equations appearing in Eq. (10) can be expressed in the form:

$$
u_{q}(r)=P(r) C_{1}^{q}+Q(r) C_{2}^{q}+R_{q}(r), \quad q=0,1,2, \ldots,
$$

where $C_{1}^{q}$ and $C_{2}^{q}$ are arbitrary constants and $P(r)$ and $Q(r)$ are the complementary functions, and

$$
R_{q}(r)=P(r) \int \frac{Q(r) H_{q}(r)}{\Delta} \mathrm{d} r-Q(r) \int \frac{P(r) H_{q}(r)}{\Delta} \mathrm{d} r,
$$

where

$$
\Delta=P(r) \frac{\mathrm{d} Q(r)}{\mathrm{d} r}-Q(r) \frac{\mathrm{d} P(r)}{\mathrm{d} r} .
$$

The nonlinear radial and hoop stresses for each case are

$$
\begin{gathered}
\sigma_{r}^{q}=\frac{E_{r}^{0}}{1-v_{r \theta} v_{\theta r}}\left[\frac{\mathrm{d} u_{q}}{\mathrm{~d} r}+\frac{1-\delta_{0 q}}{2}\left(\frac{\mathrm{d} u_{q-1}}{\mathrm{~d} r}\right)^{2}+v_{\theta r} \frac{u_{q}}{r}\right], \\
\sigma_{\theta}^{q}=\frac{E_{\theta}^{0}}{1-v_{r \theta} v_{\theta r}}\left\{v_{r \theta}\left[\frac{\mathrm{d} u_{q}}{\mathrm{~d} r}+\frac{1-\delta_{0 q}}{2}\left(\frac{\mathrm{d} u_{q-1}}{\mathrm{~d} r}\right)^{2}\right]+\frac{u_{q}}{r}\right\} .
\end{gathered}
$$

\subsection{Linear solution for some special cases}

Consider the following five cases according to the disk profiles $h(r)$ and the FG function $F(r)$. The complementary functions $P(r)$ and $Q(r)$ appearing in the solution are in the form of hypergeometric functions $F_{1,1}$ or $F_{1,2}$ in all cases. The expressions for all functions can be simplified as:

Case 1:

$$
\begin{gathered}
h=h_{0} \mathrm{e}^{-n r^{k}}, \quad F(r)=\mathrm{e}^{-m r^{k}}, P(r)=r^{\mu} F_{1,1}\left(\left[L_{11}\right],\left[L_{12}\right],(m+n) r^{k}\right), \\
Q(r)=r^{-\mu} F_{1,1}\left(\left[L_{11}-L_{12}\right],\left[2-L_{12}\right],(m+n) r^{k}\right),
\end{gathered}
$$


Case 2:

$$
\begin{gathered}
h=h_{0} \mathrm{e}^{-n r^{k}}, \quad F(r)=r^{m}, P(r)=r^{\left(\mu_{1}-m\right) / 2} F_{1,1}\left(\left[L_{21}\right],\left[L_{22}\right], n r^{k}\right), \\
Q(r)=r^{-\left(\mu_{1}-m\right) / 2} F_{1,1}\left(\left[L_{21}-L_{22}+1\right],\left[2-L_{22}\right], n r^{k}\right),
\end{gathered}
$$

Case 3:

$$
\begin{gathered}
h=h_{0}\left(1-n r^{k}\right)^{k_{1}}, F(r)=r^{m}, P(r)=r^{\left(\mu_{1}-m\right) / 2} F_{1,2}\left(\left[L_{31}, L_{32}\right],\left[L_{33}\right], n r^{k}\right), \\
Q(r)=r^{-\left(\mu_{1}-m\right) / 2} F_{1,2}\left(\left[L_{31}-L_{33}+1, L_{32}-L_{33}+1\right],\left[2-L_{33}\right], n r^{k}\right),
\end{gathered}
$$

Case 4:

$$
\begin{gathered}
h=h_{0} r^{n}, \quad F(r)=\mathrm{e}^{-m r^{k}}, P(r)=r^{\left(\mu_{2}-n\right) / 2} F_{1,1}\left(\left[L_{41}\right],\left[L_{42}\right], n r^{k}\right), \\
Q(r)=r^{-\left(\mu_{2}-n\right) / 2} F_{1,1}\left(\left[L_{41}-L_{42}+1\right],\left[2-L_{42}\right], m r^{k}\right),
\end{gathered}
$$

Case 5:

$$
h=h_{0} r^{n}, \quad F(r)=r^{m}, \quad P(r)=r^{\left(\mu_{3}+n+m\right) / 2}, \quad Q(r)=r^{\left(\mu_{3}+n+m\right) / 2} .
$$

It is to be noted that the expressions $\mu, L_{i j}$ and $\mu_{i}$ are given by

$$
\begin{gathered}
\mu=\sqrt{\frac{v_{\theta r}}{v_{r} \theta}}, \quad L_{11}=\frac{1}{k} \mu(1+\mu), \quad L_{12}=\frac{1}{k}(k+2 \mu), \quad L_{21}=\frac{1}{2 k}\left(-k m+\mu_{1}+2 \mu\right), \\
L_{22}=L_{33}=\frac{1}{k}\left(k+\mu_{1}\right), \quad L_{31}=\frac{1}{2 k}\left(k k_{1}+\mu_{1}+\mu_{4}\right), \\
L_{32}=\frac{1}{2 k}\left(k k_{1}+\mu_{1}-\mu_{4}\right), \quad L_{41}=\frac{1}{2 k}\left(-n+\mu_{2}+2 \mu_{1}\right), \quad L_{42}=\frac{1}{k}\left(k+\mu_{2}\right), \\
\mu_{i}=\sqrt{y_{i}\left(y_{i}-4 v_{\theta r}\right)+4 \mu^{2}}, \quad y_{1}=m, \quad y_{2}=n, \quad y_{3}=m+n, \quad y_{4}=m+k k_{1} .
\end{gathered}
$$

In addition, the forms of the free term $H_{q}(r)$ for the first four cases are given by

$$
H_{q}(r)=\frac{\rho^{0} \Omega^{2}}{2 E_{r}^{0}}\left(1-v_{r} v_{\theta r}\right) r
$$

while for the fifth case, it is given by

$$
H_{q}(r)=\frac{-r^{3} v_{\theta r}}{3 v_{\theta r}(m+n+3)\left[1-(m+n) v_{r \theta}\right]} .
$$

The radial and circumferential stresses for each case will be given by the substitution of $E_{r}, E_{\theta}, P(r), Q(r)$ and $R(r)$ in the corresponding equations. 


\section{Applications}

Here, we restrict our attention to the problem according to any of the above cases, namely for example the third case. The profile of the rotating disk is given in Fig. 1. In what follows, we will firstly derive the analytical elastic solution of orthotropic solid and annular disks. Moreover, the viscoelastic deformations of these disks under different conditions will be investigated.
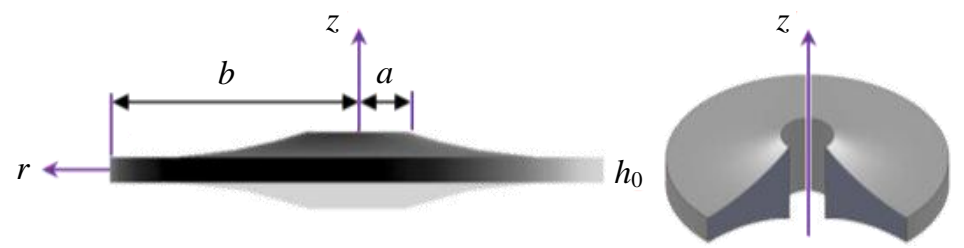

Fig. 1. Configuration of a variable-thickness annular disk of inner radius $a$ and outer radius $b$

The complementary functions $P(r)$ and $Q(r)$ appear in Eq. (21) while the free term $H_{q}(r)$ appear in Eq. (26). In these equations, $F_{i, j}(\cdot)$ are confluent hypergeometric functions. Moreover, the function $R_{q}(r)$ should appear in terms of $P(r)$ and $Q(r)$ as

$$
R_{q}(r)=\eta\left(1-v_{r \theta} v_{\theta r}\right)\left(P(r) \int \frac{r Q(r)}{\Delta} \mathrm{d} r-Q(r) \int \frac{r P(r)}{\Delta} \mathrm{d} r\right), \quad \eta=\frac{\rho^{0} \Omega^{2}}{2 E_{r}^{0}},
$$

where $\eta$ is a new parameter called the material parameter. From the above relation, the solutions of the displacement $u_{q}$ may consist of powers of the material parameter $\eta$ depending on $q$, i.e., it may take the form

$$
u_{q}(r)=\sum_{i=0}^{q} a_{i}(r) \eta^{i+1} .
$$

Suppose that $\bar{u}_{q}=u_{q} / \eta$, then one gets, after dropping the bar, the displacement and stress components as

$$
\begin{gathered}
u_{q}=\sum_{i=0}^{q} a_{i}(r) \eta^{i}, \\
\sigma_{r}^{q}=\frac{E_{r}^{0}}{1-v_{r \theta} v_{\theta r}} \sum_{i=0}^{q} \eta^{i}\left[\frac{\mathrm{d} a_{i}}{\mathrm{~d} r}+\frac{1-\delta_{0 q}}{2}\left(\frac{\mathrm{d} a_{i}}{\mathrm{~d} r}\right)^{2}+\frac{v_{\theta r}}{r} a_{i}\right], \\
\sigma_{\theta}^{q}=\frac{E_{\theta}^{0}}{1-v_{r \theta} v_{\theta r}} \sum_{i=0}^{q} \eta^{i}\left[v_{r \theta}\left(\frac{\mathrm{d} a_{i}}{\mathrm{~d} r}+\frac{1-\delta_{0} q}{2}\left(\frac{\mathrm{d} a_{i}}{\mathrm{~d} r}\right)^{2}\right)+\frac{a_{i}}{r}\right] .
\end{gathered}
$$




\subsection{Rotating annular disk}

The substitution of Eq. (14) with the aid of Eqs. (21) and (26) gives the radial and circumferential stresses in the following form:

$$
\begin{gathered}
\sigma_{r}^{q}=\frac{E_{r}^{0} r^{m}}{1-v_{r \theta} v_{\theta r}}\left[C_{1}^{q} \Phi_{1}(P, r)+C_{2}^{q} \Phi_{1}(Q, r)+\Phi_{1}\left(R_{q}, r\right)+\chi_{q}(r)\left(1-\delta_{0 q}\right)\right], \\
\sigma_{\theta}^{q}=\frac{E_{\theta}^{0} r^{m}}{1-v_{r \theta} v_{\theta r}}\left[C_{1}^{q} \Phi_{2}(P, r)+C_{2}^{q} \Phi_{2}(Q, r)+\Phi_{2}\left(R_{q}, r\right)+v_{\theta r} \chi_{q}(r)\left(1-\delta_{0 q}\right)\right] .
\end{gathered}
$$

Using the boundary conditions appearing in Eq. (11), and solving the dual algebraic equations for $C_{1}^{q}$ and $C_{2}^{q}$ gives

$$
\begin{gathered}
C_{1}^{q}=\frac{\Phi_{1}\left(R_{q}, b\right) \Phi_{1}(Q, a)-\Phi_{1}\left(R_{q}, a\right) \Phi_{1}(Q, b)+\left[\chi_{q}(b) \Phi_{1}(Q, a)-\chi_{q}(a) \Phi_{1}(Q, b)\right]\left(1-\delta_{0 q}\right)}{\Phi_{1}(P, b) \Phi_{1}(Q, a)-\Phi_{1}(P, a) \Phi_{1}(Q, b)}, \\
C_{2}^{q}=\frac{\Phi_{1}\left(R_{q}, a\right) \Phi_{1}(P, a)-\Phi_{1}\left(R_{q}, b\right) \Phi_{1}(P, a)+\left[\chi_{q}(a) \Phi_{1}(P, b)-\chi_{q}(b) \Phi_{1}(P, a)\right]\left(1-\delta_{0 q}\right)}{\Phi_{1}(P, b) \Phi_{1}(Q, a)-\Phi_{1}(P, a) \Phi_{1}(Q, b)},
\end{gathered}
$$

where

$$
\begin{aligned}
& \Phi_{1}(\psi, r)=\frac{\mathrm{d} \psi}{\mathrm{d} r}+v_{\theta r} \frac{\psi}{r}, \quad \Phi_{2}(\psi, r)=v_{\theta r} \frac{\mathrm{d} \psi}{\mathrm{d} r}+\frac{\psi}{r}, \\
& \psi \equiv\left\{P(r), Q(r), R_{q}(r)\right\}, \quad \chi_{q}(r)=\frac{1}{2}\left(\frac{\mathrm{d} u_{q-1}}{\mathrm{~d} r}\right)^{2} .
\end{aligned}
$$

\subsection{Rotating solid disk}

In the solid disk, the radial and hoop stresses should be finite at the center of the disk and the radial displacement should vanish. So, the constant $C_{2}^{q}$ should vanish. The edge of the disk is traction-free and the displacement becomes

$$
u_{q}(r)=P(r) C_{1}^{q}+R_{q}(r), \quad q=0,1,2, \ldots,
$$

in which

$$
C_{1}^{q}=\frac{\Phi_{1}\left(R_{q}, b\right)+\chi_{q}(b)\left(1-\delta_{0 q}\right)}{\Phi_{1}(P, b)} .
$$

The components of radial and circumferential stresses become

$$
\begin{aligned}
\sigma_{r}^{q} & =\frac{E_{r}^{0} r^{m}}{1-v_{r \theta} v_{\theta r}}\left[C_{1}^{q} \Phi_{1}(P, r)+\Phi_{1}\left(R_{q}, r\right)+\chi_{q}(r)\left(1-\delta_{0 q}\right)\right], \\
\sigma_{\theta}^{q} & =\frac{E_{\theta}^{0} r^{m}}{1-v_{r \theta} v_{\theta r}}\left[C_{1}^{q} \Phi_{2}(P, r)+\Phi_{2}\left(R_{q}, r\right)+v_{\theta r} \chi_{q}(r)\left(1-\delta_{0 q}\right)\right] .
\end{aligned}
$$

Then, the successive elastic nonlinear solutions for FGM solid and annular disks of arbitrary variable thickness are completed by the application of the boundary conditions. 


\subsection{Fiber-reinforced viscoelastic disks}

To deal with the viscoelastic solution, we consider a disk with viscoelastic material properties reinforced by coaxial circular elastic fibers. The method of effective moduli is used to reduce this problem to that of a FG anisotropic material with elastic coefficients $E_{r}^{0}, E_{\theta}^{0}, v_{\theta r}$ and $v_{r \theta}$. Then, one obtains (see Pobedrya [30]):

$$
\begin{gathered}
E_{r}^{0}=\frac{9 E \zeta \omega}{2(1-\gamma)+(1-\gamma+9 \gamma \zeta) \omega}=\frac{9 E \zeta}{2(1-\gamma) \beta_{2}}\left[1-g_{\beta_{2}}(\omega)\right], \\
E_{\theta}^{0}=E\left[\gamma+9 \zeta(1-\gamma) \frac{\omega}{2+\omega}\right]=E\left\{\gamma+9 \zeta(1-\gamma)\left[1-g_{\beta_{1}}(\omega)\right]\right\}, \\
v_{\theta r}=\gamma \nu+(1-\gamma) \frac{1-\omega}{2+\omega}=\gamma \nu-(1-\gamma)\left[1-\frac{3}{2} g_{\beta_{1}}(\omega)\right],
\end{gathered}
$$

and

$$
\zeta=\frac{K}{E}, \quad \beta_{1}=\frac{1}{2}, \quad \beta_{2}=\frac{1}{2}\left(1+\frac{9 \gamma \zeta}{1-\gamma}\right), \quad g_{\beta_{i}}(\omega)=\frac{1}{1+\beta_{i} \omega}, \quad i=1,2 .
$$

The other Poisson's ratio $v_{r \theta}$ may be easily obtained using Eqs. (42)-(44) such that Eq. (6) should be satisfied.

In what follows, we consider the radial and hoop stresses and radial displacement to be functions of the parameters $\omega, E, v, \gamma$ and $\zeta$ in elastic composite disks and may be operators of time $t$ in viscoelastic composite disks. Generally, the radial displacement $u(r)$ can be represented according to Illyushin's approximation method of unified form (Allam and Pobedrya [31] and Illyushin and Pobedrya [32]):

$$
u(r, \omega)=\sum_{i=1}^{5} A_{i}(r) \phi_{i}(\omega)
$$

where

$$
\phi_{1}=1, \quad \phi_{2}=\omega, \quad \phi_{3}=\pi=\frac{1}{\omega}, \quad \phi_{4}=g_{\beta_{1}}(\omega), \quad \phi_{5}=g_{\beta_{2}}(\omega),
$$

in which $\beta_{j}$ and $g_{\beta_{j}}(\omega), j=1,2$, have appeared in Eq. (45).

Equation (46) contain the coefficients $A_{i}, i=1,2, \ldots, 5$ as functions of $r, \omega$, $v, \gamma$, and $\zeta$. These coefficients may be found from the system of algebraic linear equations

$$
\sum_{j=1}^{5} \psi_{i j} A_{j}=B_{i}
$$

in which

$$
\psi_{i j}=\int_{0}^{1} \phi_{i}(\omega) \phi_{j}(\omega) \mathrm{d} \omega, \quad B_{i}=\int_{0}^{1} u(r, \omega) \phi_{i}(\omega) \mathrm{d} \omega, \quad i, j=1,2, \ldots, 5 .
$$


The relaxation function $\omega(t)$ may be considered in the form

$$
\omega(t)=b_{1}+b_{2} \mathrm{e}^{-\alpha t}
$$

where $b_{1}, b_{2}$ and $\alpha$ are constants. We use Laplace-Carson transformation to determine the functions $\pi(t)$ and $g_{\beta_{j}}(t)$. Setting their transformations by $\pi^{*}=1 / \omega^{*}$ and $g_{\beta_{j}}^{*}$, we get

$$
\omega^{*}(s)=b_{1}+\frac{s}{s+\frac{1}{\alpha}} b_{2} .
$$

Thus

$$
\begin{gathered}
\pi(t)=\frac{1}{b_{1}}\left[1-b_{2} \mathrm{e}^{b_{1}\left(b_{1}+b_{2}\right) \tau}\right], \tau=\alpha t \\
g_{\beta_{j}}(t)=\frac{1}{1+\beta_{j} b_{1}}\left[1-\frac{\beta_{j} b_{2} \mathrm{e}^{-\left(\frac{\left(1+\beta_{j} b_{1}\right) \tau}{1+\beta_{j}\left(b_{1}+b_{2}\right)}\right)}}{1+\beta_{j}\left(b_{1}+b_{2}\right)}\right], j=1,2 .
\end{gathered}
$$

Equation (46) for a viscoelastic composite disk may be decoded to obtain explicit formulae for field quantities in terms of the time parameter $t$. First, we have for the radial displacement

$$
\begin{aligned}
u(r, t)= & A_{1}(r) \Omega^{2}(t)+A_{2}(r) \int_{0}^{t} \omega(t-\tau) \mathrm{d} \Omega^{2}(\tau)+A_{3}(r) \int_{0}^{t} \pi(t-\tau) \mathrm{d} \Omega^{2}(\tau) \\
& +A_{4}(r) \int_{0}^{1} g_{\beta_{1}}(t-\tau) \mathrm{d} \Omega^{2}(\tau)+A_{5}(r) \int_{0}^{1} g_{\beta_{2}}(t-\tau) \mathrm{d} \Omega^{2}(\tau)
\end{aligned}
$$

Putting

$$
\Omega^{2}(t)=\Omega_{0}^{2} H(t),
$$

where $H(t)$ is Heaviside's unit step function

$$
H(t)= \begin{cases}0 & t<0 \\ 1 & t>0\end{cases}
$$

Then

$$
u(r, t)=\Omega_{0}^{2}\left[A_{1} H(t)+A_{2} \omega(t)+A_{3} \pi(t)+A_{4} g_{\beta_{1}}(t)+A_{5} g_{\beta_{2}}(t)\right],
$$

where $\omega(t), \pi(t)$ and $g_{\beta_{i}}(t)$ are given in Eqs. (50) and (52). Using the same technique as mentioned in Eq. (56) with the aid of Eqs. (4), we can obtain the radial and hoop stresses for the rotating viscoelastic FG variable-thickness solid and annular disks. 


\section{Numerical examples and discussions}

The numerical examples for the rotating FG fiber-reinforced viscoelastic disks with gradually varying thickness will be discussed. The results of the present disks are plotted in Figs. 2-7. The values of coefficients $b_{1}$ and $b_{2}$ (firstly appearing in Eq. (50)) were taken to be 0.1 and 0.9 , respectively, and $v=0.3$ while the coefficient $\alpha$ depends on the scale of measuring the time, the time parameter $\tau(\equiv \alpha t)$ is given in terms of it.

The constitutive parameter is chosen to be $\zeta \equiv(K / E)=0.5$. Also, the geometric parameters $n, k, k_{1}$ are considered such that $n=0.4, k=0.7$, and $k_{1}=1.2$ and the FG coefficient $m=0.3$. Also, the parameters $\omega$ and $\eta$ are fixed, except otherwise stated, as $\omega=0.5$ and $\eta=0.4$. In addition, the inner radius to outer radius ratio is fixed to be $s \equiv(a / b)=0.1$.

Fig. 2(a,b) is performed for viscoelastic FG annular and solid disks with gradually varying thickness. For annular disk, the radial stress $\sigma_{r}$ has its minimal zero value at the edges to coincide with the boundary conditions. The maximum value of $\sigma_{r}$ may occur at $r=0.4$. The hoop stress $\sigma_{\theta}$ decreases as $r$ increases along the radial direction. It starts with a maximum value at the inner edge and ends with a smallest value at the outer edge. However, the radial displacement $u$ decreases as $r$ increases along the radial direction of the disk. It starts with a smallest value at the inner edge and ends with a maximum value at the outer edge of the disk. For solid disk, the radial stress $\sigma_{r}$ starts with a constant value at the inner edge. It is no longer increasing and has its maximum value at $r=0.4$. Then, it decreases rapidly to get zero value at the outer edge. The circumferential stress $\sigma_{\theta}$ starts with a small

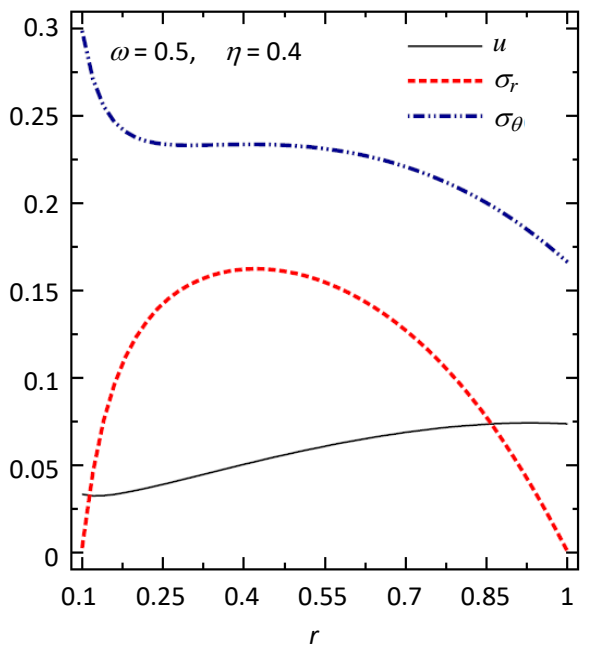

(a) Annular disk

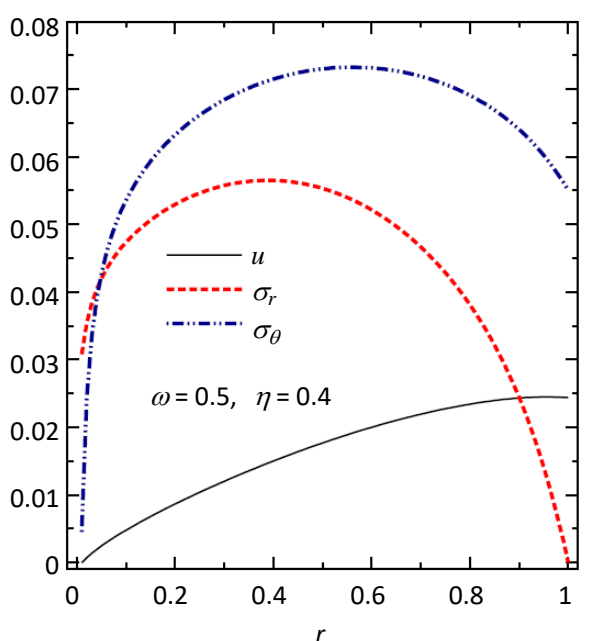

(b) Solid disk

Fig. 2. Displacement $u$, radial stress $\sigma_{r}$ and circumferential stress $\sigma_{\theta}$ in the variable-thickness annular and solid disks 
value at the inner edge. It is no longer increasing and has its maximum at $r=0.55$. The radial displacement $u$ vanish at the inner edge and gives its highest value at the outer edge. It may be linearly increases along the radial direction.

Figs. 3-5 show the displacements and stresses versus $r$ with different values of the approximation order number $q$. Fig. 3 shows that for the annular disk the displacement $u$ is slightly sensitive to the variation of $q$. It just increases as $r$ increases. The values of $u$ for $q \neq 0$ may be less than the corresponding one when $q=0$ and this occurs at outer edge of the disk. This is not the same for the solid disk in which the displacement $u$ is very sensitive to the variation of the approximation order number $q$. In fact, the higher values of $q$ gives appropriate radial displacement.

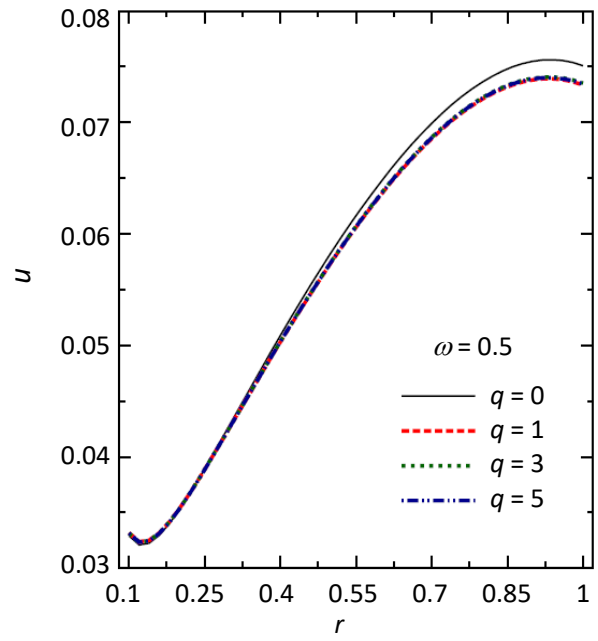

(a) Annular disk

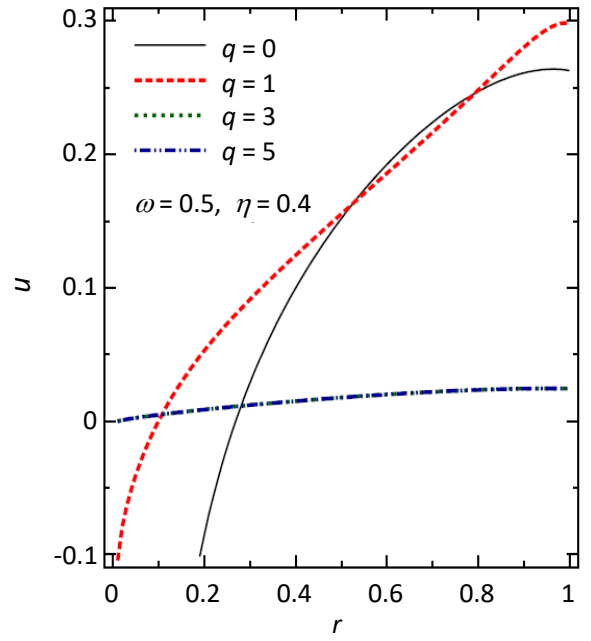

(b) Solid disk

Fig. 3. Distribution of radial displacement $u$ along the radial direction of the variable-thickness annular and solid disks

Fig. 4 shows that for the annular disk the radial stress $\sigma_{r}$ is lightly sensitive to the variation of $q$. It has its maximum value at $r=0.4$ and satisfies the boundary conditions. Also, this is not the same for the solid disk in which the radial stress $\sigma_{r}$ is very sensitive to the variation of the approximation order number $q$. In fact, $q=0$ and $q=1$ are not suitable to give accurate radial stresses.

Fig. 5 shows that for the annular disk the circumferential stress $\sigma_{\theta}$ is lightly sensitive to the variation of $q$. It is decreasing as $r$ increases. The values of $\sigma_{\theta}$ due to $q \neq 0$ may be less than the corresponding one when $q=0$ and this occurs when $r>0.3$. This is not the same for the solid disk in which the circumferential stress $\sigma_{\theta}$ is very sensitive to the variation of the approximation order number $q$. As mentioned above, the higher values of $q$ gives appropriate circumferential stress. 


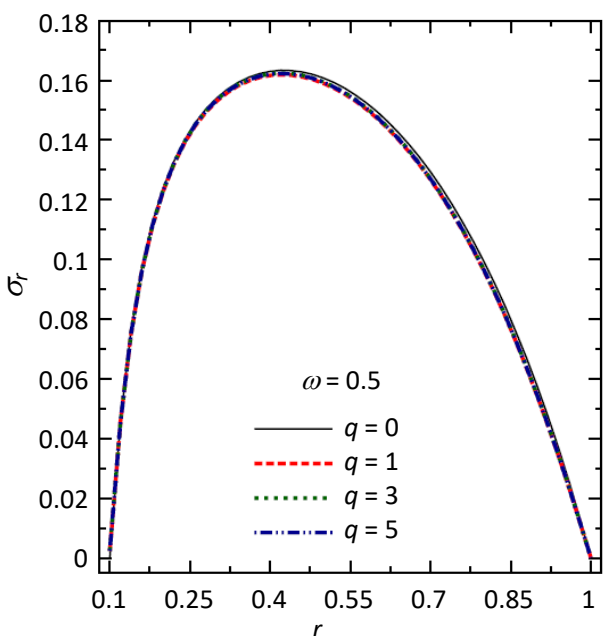

(a) Annular disk

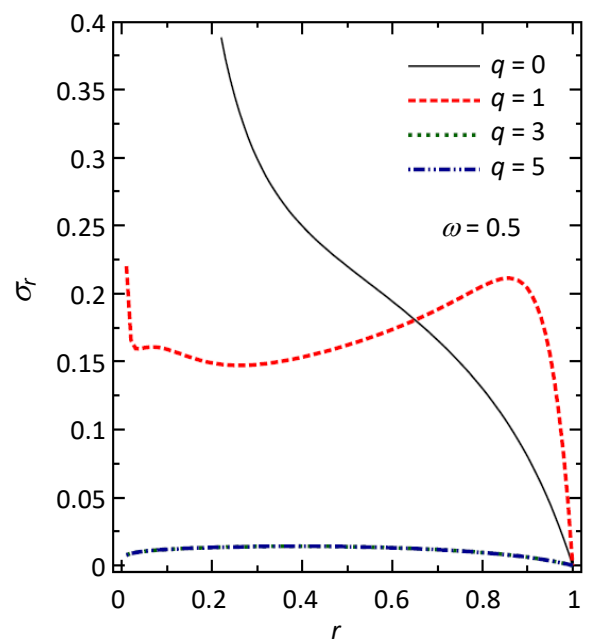

(b) Solid disk

Fig. 4. Distribution of radial stress $\sigma_{r}$ along the radial direction of the variable-thickness annular and solid disks

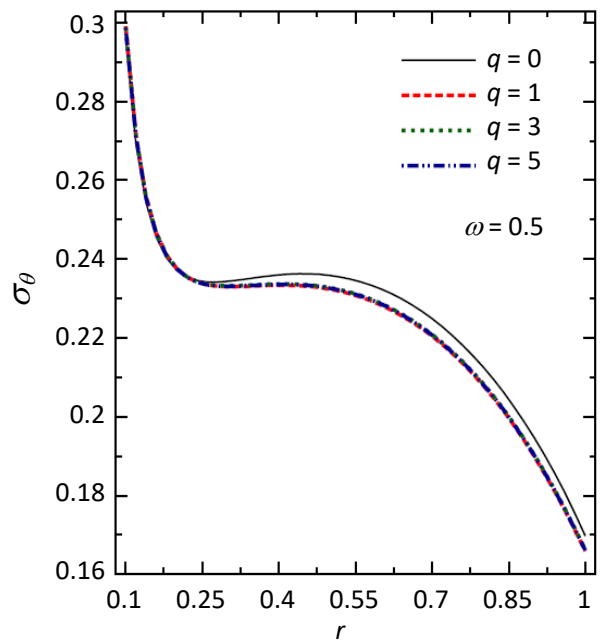

(a) Annular disk

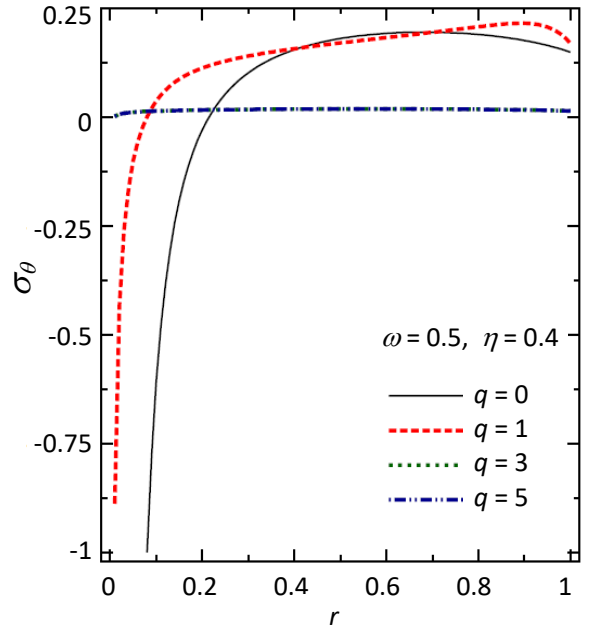

(b) Solid disk

Fig. 5. Distribution of circumferential stress $\sigma_{\theta}$ along the radial direction of the variable-thickness annular and solid disks

Fig. 6 shows the plots of the radial displacement and radial and hoop stresses of the rotating annular and solid disks at different positions. The influence played by the time parameter on the field quantities is investigated. For annular disk, the radial displacement $u$ increases as the time parameter $\tau$ increases while it decreases as $\tau$ increases for the solid disk. Also, both $\sigma_{r}$ and $\sigma_{\theta}$ are decreasing with the increasing 


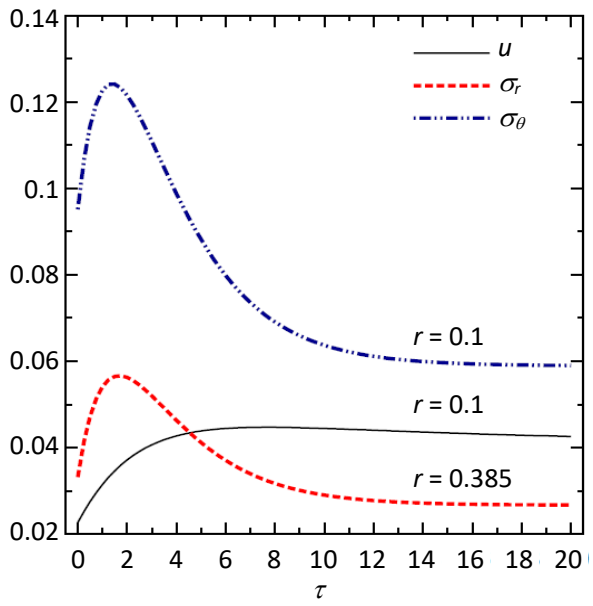

(a) Annular disk

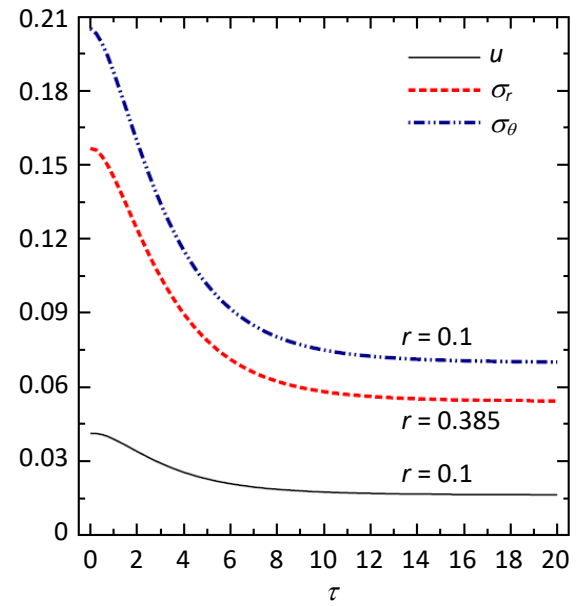

(b) Solid disk

Fig. 6. Displacement $u$, radial stress $\sigma_{r}$ and circumferential stress $\sigma_{\theta}$ versus $\tau$ in the variable-thickness annular and solid disks

of $\tau$ for the solid disk. However, for the annular disk, the two stresses are no longer increasing and have their maximum value at the same time parameter $\tau=1.7$. All displacement and stresses still unchanged for $\tau>10$.

Finally, the effect of the material parameter $\eta$ which appears in Eq.(28) on the distribution of $u, \sigma_{r}$ and $\sigma_{\theta}$ is investigated in Fig. 7. For the annular disk are plotted versus $\eta$ at the same position $r=0.385$ while for the solid disk they plotted

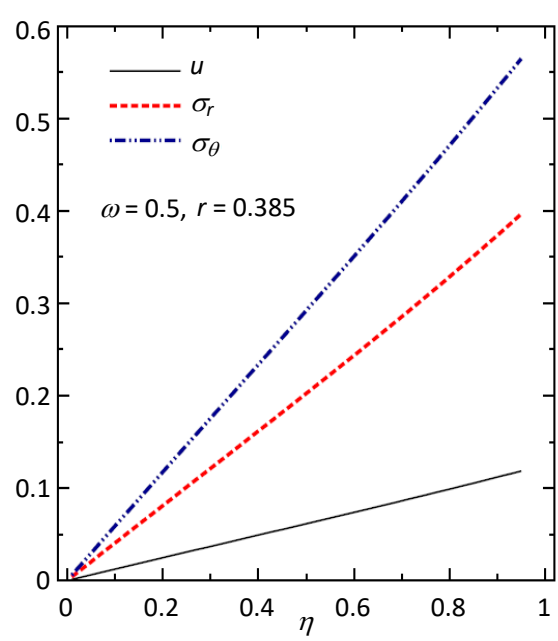

(a) Annular disk

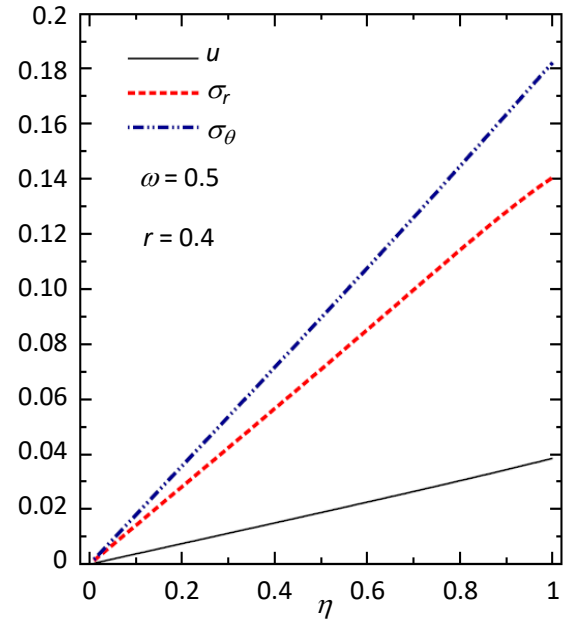

(b) Solid disk

Fig. 7. Displacement $u$, radial stress $\sigma_{r}$ and circumferential stress $\sigma_{\theta}$ versus $\eta$ in the variable-thickness annular and solid disks 
at $r=0.4$. For both disks the displacement and stresses are linearly changed wilt the material parameter $\eta$. The values of all fields for the annular disk are the highest ones.

\title{
6. Conclusions
}

Rotating viscoelastic inhomogeneous composite disks with gradually varying thickness, density and material properties is treated herein. This problem is firstly solved by using the method of successive approximation then by using the methods of effective moduli and Illyushin's approximation. It is of interest to select some examples that illustrate the essential features of the present problem. Five solutions for five problems may be treated in this work. The analytical elastic and viscoelastic solution have been obtained for the rotating FG solid and annular disks with gradually varying thickness. In addition, the analytical elastic solution of rotating uniform-thickness disks is obtained as special case of the present problem. For the sake of completeness and comparison, some examples are illustrated. The effects due to approximation order number, material parameter and time parameter on the displacement and stresses are investigated.

\author{
Manuscript received by Editorial Board, July 07, 2017; \\ final version, November 06, 2017.
}

\section{References}

[1] S. Tang. Elastic stresses in rotating anisotropic disks. International Journal of Mechanical Sciences, 11(6):509-517, 1969. doi: 10.1016/0020-7403(69)90052-6.

[2] A.C. Ugural and S.K. Fenster. Advanced Strength and Applied Elasticity. Elsevier, 1987.

[3] A.N. Eraslan and Y. Orcan. Elastic-plastic deformation of a rotating solid disk of exponentially varying thickness. Mechanics of Materials, 34(7):423-432, 2002. doi: 10.1016/S01676636(02)00117-5.

[4] A.N. Eraslan and H. Argeso. Limit angular velocities of variable thickness rotating disks. International Journal of Solids and Structures, 39(12):3109-3130, 2002. doi: 10.1016/S00207683(02)00249-4.

[5] S.P. Timoshenko and J.N. Goodier. Theory of Elasticity. McGraw Hill, New York, 1970.

[6] C.O. Horgan and A.M. Chan. The stress response of functionally graded isotropic linearly elastic rotating disks. Journal of Elasticity, 55(3):219-230, 1999. doi: 10.1023/A:1007644331856.

[7] H. Jahed, B. Farshi, and J. Bidabadi. Minimum weight design of inhomogeneous rotating discs. International Journal of Pressure Vessels and Piping, 82(1):35-41, 2005. doi: 10.1016/j.ijpvp.2004.06.006.

[8] M.H. Hojjati and A. Hassani. Theoretical and numerical analyses of rotating discs of non-uniform thickness and density. International Journal of Pressure Vessels and Piping, 85(10):694-700, 2008. doi: 10.1016/j.ijpvp.2008.02.010.

[9] A.N. Eraslan and T. Akis. On the plane strain and plane stress solutions of functionally graded rotating solid shaft and solid disk problems. Acta Mechanica, 181(1):43-63, 2006. doi: 10.1007/s00707-005-0276-5. 
[10] S.A.H. Kordkheili and R. Naghdabadi. Thermoelastic analysis of a functionally graded rotating disk. Composite Structures, 79(4):508-516, 2007. doi: 10.1016/j.compstruct.2006.02.010.

[11] L.H. You, X.Y. You, J.J. Zhang, and J. Li. On rotating circular disks with varying material properties. Zeitschrift für Angewandte Mathematik und Physik (ZAMP), 58(6):1068-1084, 2007. doi: 10.1007/s00033-007-5094-2.

[12] M.H. Hojjati and S. Jafari. Semi-exact solution of elastic non-uniform thickness and density rotating disks by homotopy perturbation and Adomian's decomposition methods. Part I: Elastic solution. International Journal of Pressure Vessels and Piping, 85(12):871-878, 2008. doi: 10.1016/j.ijpvp.2008.06.001.

[13] V. Vullo and F. Vivio. Elastic stress analysis of non-linear variable thickness rotating disks subjected to thermal load and having variable density along the radius. International Journal of Solids and Structures, 45(20):5337-5355, 2008. doi: 10.1016/j.jjsolstr.2008.05.018.

[14] F. Vivio and V. Vullo. Elastic stress analysis of rotating converging conical disks subjected to thermal load and having variable density along the radius. International Journal of Solids and Structures, 44(24):7767-7784, 2007. doi: 10.1016/j.jisolstr.2007.05.013.

[15] M. Bayat, M. Saleem, B.B. Sahari, A.M.S. Hamouda, and E. Mahdi. Analysis of functionally graded rotating disks with variable thickness. Mechanics Research Communications, 35(5):283309, 2008. doi: 10.1016/j.mechrescom.2008.02.007.

[16] M. Bayat, M. Saleem, B.B. Sahari, A.M.S. Hamouda, and E. Mahdi. Mechanical and thermal stresses in a functionally graded rotating disk with variable thickness due to radially symmetry loads. International Journal of Pressure Vessels and Piping, 86(6):357-372, 2009. doi: 10.1016/j.ijpvp.2008.12.006.

[17] M. Bayat, B.B. Sahari, M. Saleem, A. Ali, and S.V. Wong. Thermoelastic solution of a functionally graded variable thickness rotating disk with bending based on the first-order shear deformation theory. Thin-Walled Structures, 47(5):568-582, 2009. doi: 10.1016/j.tws.2008.10.002.

[18] M. Bayat, B.B. Sahari, A. Saleem, M .and Ali, and S.V. Wong. Bending analysis of a functionally graded rotating disk based on the first order shear deformation theory. Applied Mathematical Modelling, 33(11):4215-4230, 2009. doi: 10.1016/j.apm.2009.03.001.

[19] A.M. Zenkour. Elastic deformation of the rotating functionally graded annular disk with rigid casing. Journal of Materials Science, 42(23):9717-9724, 2007. doi: 10.1007/s10853-0071946-6.

[20] A.M. Zenkour. Stress distribution in rotating composite structures of functionally graded solid disks. Journal of Materials Processing Technology, 209(7):3511-3517, 2009. doi: 10.1016/j.jmatprotec.2008.08.008.

[21] G.J. Nie and R.C. Batra. Stress analysis and material tailoring in isotropic linear thermoelastic incompressible functionally graded rotating disks of variable thickness. Composite Structures, 92(3):720-729, 2010. doi: 10.1016/j.compstruct.2009.08.052.

[22] W.W. Feng. On finite deformation of viscoelastic rotating disks. International Journal of Non-Linear Mechanics, 20(1):21-26, 1985. doi: 10.1016/0020-7462(85)90044-7.

[23] A.M. Zenkour and M.N.M. Allam. On the rotating fiber-reinforced viscoelastic composite solid and annular disks of variable thickness. International Journal for Computational Methods in Engineering Science and Mechanics, 7(1):21-31, 2006. doi: 10.1080/155022891009639.

[24] M.N.M. Allam, A.M. Zenkour, and T.M.A. El-Azab. Viscoelastic deformation of the rotating inhomogeneous variable thickness solid and annular disks. International Journal for Computational Methods in Engineering Science and Mechanics, 8(5):313-322, 2007. doi: 10.1080/15502280701471657.

[25] A.M. Zenkour. Thermoelastic analysis of an annular sandwich disk with metal/ceramic faces and functionally graded core. Journal of Thermoplastic Composite Materials, 22(2):163-181, 2009. doi: 10.1177/0892705708091770. 
[26] A.M. Zenkour. Analytical solutions for rotating exponentially-graded annular disks with various boundary conditions. International Journal of Structural Stability and Dynamics, 5(04):557577, 2005. doi: 10.1142/S0219455405001726.

[27] A.M. Zenkour. Thermoelastic solutions for annular disks with arbitrary variable thickness. Structural Engineering and Mechanics, 24(5):515-528, 2006. doi: 10.12989/sem.2006.24.5.515.

[28] A.M. Zenkour. Steady-state thermoelastic analysis of a functionally graded rotating annular disk. International Journal of Structural Stability and Dynamics, 6(04):559-574, 2006. doi: 10.1142/S0219455406002064.

[29] A.M. Zenkour. Rotating moderately thick annular disks via an extension to classical theory. Journal of Mechanics, 28(2):355-360, 2012. doi: 10.1017/jmech.2012.39.

[30] B.E. Pobedrya. Structural anisotropy in viscoelasticity. Polymer Mechanics, 12(4):557-561, 1976. doi: 10.1007/BF00857005.

[31] M.N.M. Allam and B.E. Pobedrya. On the solution of quasi-statical problems of anisotropic viscoelasticity. Mekhanica, 31:19-27, 1978. (in Russian).

[32] A.A. Illyushin and B.E. Pobedrya. Foundations of Mathematical Theory of Thermo-ViscoElasticity. Nauka, Moscow, 1970. (in Russian). 